\title{
Probabilistic Assessment of Road Risks for Improving Logistics Processes
}

\author{
Abdelaziz Lakehal ${ }^{1, *}$, and Fouad Tachi $^{2}$ \\ ${ }^{1}$ Department of Mechanical Engineering, Mohamed Chérif Messaadia University, P.O. Box 1553, \\ Souk-Ahras, 41000, Algeria. e-mail: lakehal21@yahoo.fr \\ ${ }^{2}$ Sonatrach, Algerian Petroleum Institute, School of Skikda, Algeria
}

\begin{abstract}
The Intermodal transport represents a solution, which has proved its effectiveness, for the supply of the various logistic platforms. Road transport is also one of the means of transport used in the logistic function and is the most common. This type of transport is especially recommended for medium and short distance journeys. Transport is an important link in the logistical chain. Several constraints accompany this transport function such as: delays, flexibility, diversity of merchandise, and road risks. To identify this last problem of road risk and to minimize its influence, a Bayesian network has been developed in this paper. Through experts' surveys and research in the literature, the various risks were identified. The structure of the Bayesian network is defined on the basis of this census. The network settings vary from one situation to another. The exploitation of statistics and historical files of the transport company has allowed to define the parameters (probabilities) given in the example studied in this paper.

To prevent risks and anticipate failures in the logistics function, while optimizing a utility function, an influence diagram was used. This tool has provided the ability to control actions and make decisions safely. An example of merchandise transport between two port companies has shown promising results and better efficiency in the anticipation of actions.
\end{abstract}

\section{Introduction}

The number of logistic platforms continues to grow. Also, demand on merchandise continues to increase. Movement of merchandise within and outside a company is part of the logistic function. Ensuring logistics between multiple platforms is an activity that needs to be well managed and never left to chance. Managing logistics means organizing, executing and controlling the movement of merchandise. Several synthesis works, prepared by experts, have proposed techniques and means whose purpose is to ensure handling movements, transport, supply, deliveries, and management of the production flows, while respecting the deadlines and costs.

In this context, transport and logistics are part of the same activity. To understand and analyze the impact of failures in merchandise transport business on the logistic function,

\footnotetext{
* Corresponding author: lakehal21@yahoo.fr
} 
several studies have been carried out in recent years [1, 2]. Road transport ensures merchandise serving at long distance. Maximum permissible rolling load (from 40 to $60 \mathrm{t}$ according to countries) is regulated [3]. The road mode is today the dominant mode of transport with nearly $80 \%$ of market share. Containers are transported by trucks carrying either one or two containers. The transport of bulk merchandise has its specific risks.

Mathematical modeling, operational research and simulation are widely used tools to meet needs for risk control and optimization of the logistic function. Caution should be taken when projecting the results of these studies on real cases. Even if the results are close to reality, real experiences always hide unforeseen events. Road network architecture and drivers behavior have a big influence on the number of accidents that already occurred [4]. Also the lack of visibility favors the occurrence of road accidents, real-time prediction systems have been developed in recent years [5].

Current research works focus on developing means to eliminate these risks. The way managers prefer to manage these risks is another issue, do they prefer to manage them independently, or do they prefer to manage these risks with their industrial partners in a collaborative approach? Here, the permanent concern is to anticipate the impact of taken actions, and before that, what decision must be taken to optimize the logistic function?

To answer these questions, our study will seek to understand, analyze, and control the risks associated with the logistic function. To do this, and as the answer to all these questions is formulated as a decision problem, we will inventory, prioritize, and quantify these risks through a Bayesian network, and treat the decision issue with an influence diagram, while optimizing a utility function measured by the satisfaction.

Through this article, we will see that road safety is a complex subject since the factors defining the risk are: the human factor, the information, the structure and the reliability of the vehicles. This article does not pretend to solve all the difficulties related to determining the probabilities of road risk causes, but provides useful information so that logistics practitioners can identify their risks and avoid methodological errors, if they are asked to conduct an analysis on the subject. From a probabilistic point of view, the Bayesian networks, developed later in this paper, have the effect of reducing the probability of the variable affecting merchandise delivery with conformity and on schedule. Indeed, the most penalizing undesirable phenomenon can occur only if one or several causes take place.

\section{Research Tools for Probabilistic Reasoning}

Bayesian networks and influence diagrams [6] are probabilistic graphical models; they are also called belief networks and causal networks. These models were initially used in medical field and subsequently in industry, where they gave encouraging results especially for diagnosis, classification, failure prediction, and risk analysis [7-9]. These models are characterized by their ability to process uncertain information and to represent the interdependencies between different variables of a given problem. Their main advantage lies in their simple and understandable graphic representation, even by a non specialist. Moreover, it is known that probabilistic risk analysis has many advantages because it allows evaluating the probability of failure of a complex system, so that its weak points can be identified. Another advantage of probabilistic graphical models concerns the representation of complex and dynamic systems. Object-oriented Bayesian networks and dynamic Bayesian networks are extensions of Bayesian networks that deal with the dynamic behavior of large systems. In this article, Netica software [10] is used to build an analysis and road risk assessment model to anticipate failures in the logistics function. 


\subsection{Bayesian belief networks tool}

Like all artificial intelligence techniques, Bayesian networks require inputs. On the basis of these input data and by inference, it is possible to calculate the outputs. In the form of probabilities, these output data are represented by probability distributions. An a posteriori probability is the conditional probability on the collected data, by combination of a priori probability and likelihood via the Bayes theorem [11]. The relationship between variables is defined by a conditional probability table.

A conditional probability table is always associated with the child node. This table quantifies the effect of parent nodes on the child node. It describes the probabilities associated with the child nodes according to the different values of the parent nodes. Root nodes are defined by a priori probabilities and do not have a conditional probability table.

Child to parent dependencies, are not allowed in Bayesian networks. Therefore, the variables and arcs constituting the network will form an oriented acyclic graph.

Formally, a BN [12] is defined by:

- an acyclic oriented graph $G, G=(V, E)$, where $V$ is the set of nodes of $G$, and $E$ is the set of arcs of G,

- a probabilistic space $(\Omega, Z, P)$, with a non-empty finite set, Z a set of subspaces of $\Omega$, and $\mathrm{P}$ a probability measure on $\mathrm{Z}$ with $\mathrm{P}(\Omega)=1$,

- a set of random variables associated with the nodes of the graph $\mathrm{G}$ and defined on $(\Omega$, Z, P), such that:

$$
P\left(V_{1}, V_{2}, \ldots, V_{x a}\right)=\prod_{t=1}^{n} P\left(V_{t} / c\left(V_{t}\right)\right)
$$

Where $\mathrm{C}(\mathrm{Vi})$ is the set of parents (or causes) of Vi in the graph $\mathrm{G}$.

\subsection{Influence diagrams tool}

The solution to a decision problem is to determine an optimal strategy, which optimizes the expected utility for the decision maker, and to calculate the optimal expected utility to adhere to this strategy. An influence diagram is a probabilistic network for reasoning about decision making under uncertainty. It is a graphical representation of a decision problem involving, in an interleaved order, decisions and observations. It also represents a quantification of the strengths and / or weaknesses of the decision-maker's dependency relationships and preferences. An influence diagram can be considered as a Bayesian network to which are added decision variables and utility functions indicating the decision maker's preferences (Fig. 1).

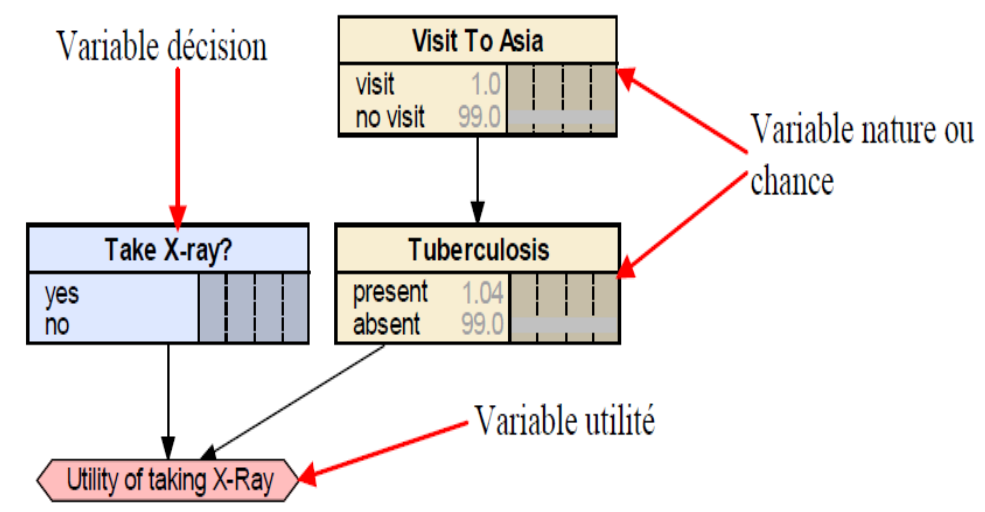

Fig. 1. Nodes types generated with Netica software. 
Three types of variables are to be considered for the influence diagrams: chance nodes, decision nodes and utility nodes. Preference is very important in decision making. It can be based on an objective quantity or on a quantity that has no numerical measure, customer satisfaction for example. The probabilistic inference in influence diagrams involves propagating the evidence observed in the graph, by calculating the a posteriori probabilities of the hidden variables, according to the values of the observed variables, and by obtaining the value of the utility for each possible decision.

\section{Road Transport Risks Assessment}

Logistic performance is a multi-criteria variable, the most traditional being: cost, quality, reliability, deadlines, and information. The new features of the logistic information system should not overshadow the traditional functionalities that provide support for activity forecasting, logistic devices design, and operations planning and conduct. Transport is one of the logistic activities that requires an information system as modern as possible. The transport profession includes land transport (roads and rails), waterway-transport (maritime and rivers), and air transport.

Road transport of merchandise is one of the main families of transport professions. The volumes of road transport of merchandise have increased significantly in recent years. This evolution is accompanied by an increase change in risks. What is needed to control the risks associated with this mode of transport is to define a risk assessment indicator, with an evaluation mechanism to measure the overall safety of a road transport mission. Good indicators can provide better information on the level of risk. Use a $\mathrm{BN}$ to analyze and diagnose the various risks associated with road transport in order to identify and quantify the most important indicators, and to determine the relationships between these indicators (causes-consequences). Data obtained from survey and experts evaluation represent the a priori probabilities for the $\mathrm{BN}$ nodes.

\subsection{Problem formulation}

\subsubsection{Establishment of the Bayesian network structure}

The action plan to prevent road risk in the company is based on the highlighted risks. A part of the actions to be implemented concerns the management and organization of human and material resources. Preventing road risks consists to act on [13]:

- Displacements and infrastructure

- Vehicles and maintenance

- Communications during displacements

- Skills and training

Several risks are associated with road transport. If personal injury risks are the most significant, others, such as those related to driving activity, are to be considered. The variables listed above are the input variables in the Bayesian model. By projection of intuitive reasoning and using graph theory, it is possible to model the causality between risks and the variable „merchandise supply” taken as a variable representing the logistic function. The Bayesian network, given in Fig. 2, facilitates the dialogue between modeler (expert) and user (Manager / Business Leader). 


\subsubsection{Example modeling}

In this paper, we deal with the case of a supply of goods between two harbor companies in Algeria, the harbor company of Algiers, EPA (capital of Algeria), and the harbor company of Jijel, EPJ (located in eastern Algeria). Through an evaluation of the risks related to the transport of goods, we evaluate the influence of these risks on the supply activity.

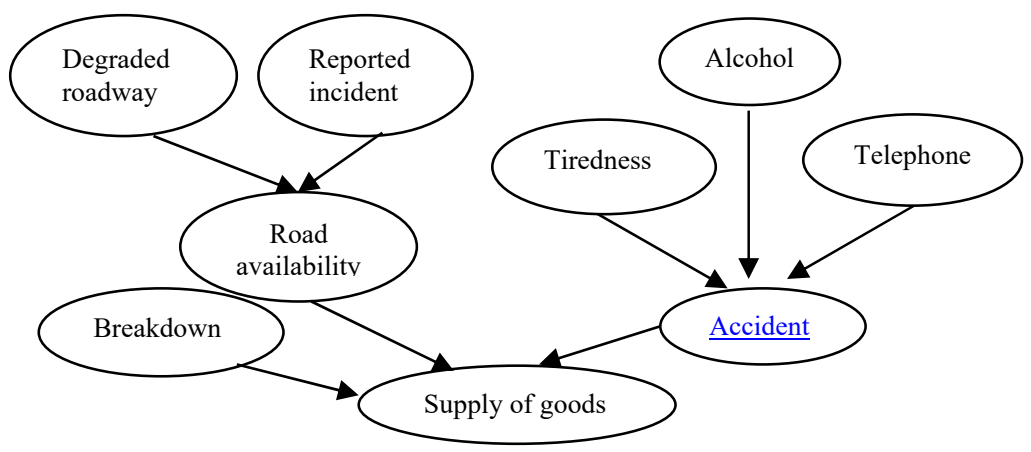

Fig. 2. Causal network modeling the influence of different risks on supply.

\section{A. Definition of the Bayesian network parameters}

Network parameters are given by the a priori probabilities of each input variable or node (see Table1.). The data processed in this case study are binary true or false.

Goods are transported by semi-trailer traveling east-west between EPA and EPJ over a distance of $241.91 \mathrm{~km}$, of which $34.11 \mathrm{~km}$ represents a degraded segment.

Table 1. Input probabilities for the Bayesian model

\begin{tabular}{ll}
\hline Variable & A priori Probability \\
\hline Telephone & Yes at $95 \%$ - No at $5 \%$ \\
Alcohol & True at $2 \%$ - False at $98 \%$ \\
Tiredness & True at $15 \%$ - False at $85 \%$ \\
Reported incident & yes at $50 \%-$ No at $50 \%$ \\
Degraded roadway & Good at $85.9 \%$ - Bad at $14.1 \%$ \\
Breakdown & True at $22 \%$ - False at $78 \%$ \\
\hline
\end{tabular}

\subsection{A posteriori probabilities calculation}

Graph and probability theories are at the origin of Bayesian networks. In this context, the construction of the Bayesian network, in Fig. 3, is essentially based on the definition of its graphical structure and probabilistic space. Mainly the a priori probabilities are the inputs of the model. The calculation phase gives rise to the definition of the a posteriori probabilities. This phase is called inference in the Bayesian approach; it consists of calculating conditional probabilities of events connected to each other by causal relationships. The inference, in a network of causalities, consists of propagating one or more information within this network, to deduce how the beliefs concerning the other nodes are modified. Fig. 3 shows the Bayesian network that models the influence of road risks on the merchandise supply. This network is based on the Netica software. In case there is no prior information on the variable, the value of 0.5 will be given, by the software, to its probability. 


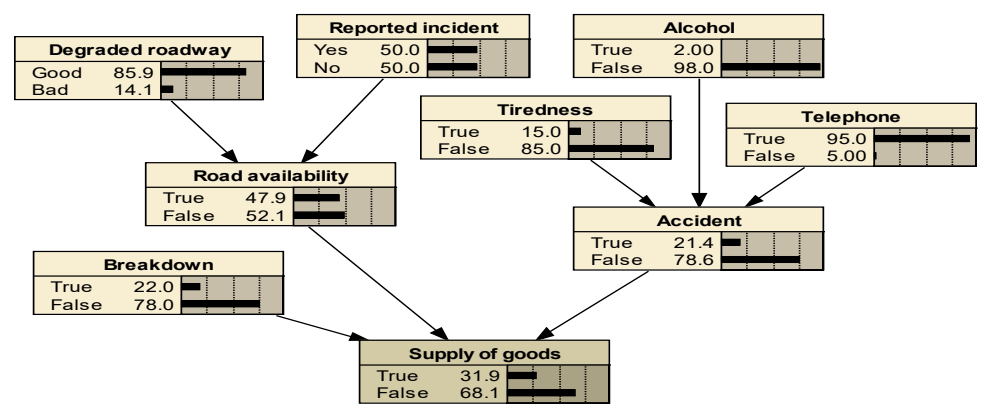

Fig. 3. BN modeling the influence of different risks on supply.

\section{Inference Results Analysis}

The independence between the variables of the network (Fig. 3) is very clear. Therefore, the inference becomes simple and easy. Also the a posteriori information, on the supply, will be available after inference in this network. A probability that the supply will be insured by $21.4 \%$ is not acceptable by the majority of companies active in logistics field. But if we return back to the causes of this high probability and by analysis of the network of Fig. 3, we notice that the availability of the road and the occurrence of an accident are the two variables that most influence the supply variable. Now by reasoning and reverse reading in the network of Fig. 3, it is possible to deduce that telephone communications are the main cause of road accidents. Strong beliefs in one cause at the expense of another change the results of the inference. For the case study presented in this article, the a priori probabilities of the declared incident variable are all equal, since there is no a priori information on the events that occurred on the road, but the strong belief in roadway degradation has not strongly influenced the probability of road availability.

Once the a posteriori probabilities are calculated, the corrective actions can be defined. In a certain reasoning environment and based on the results found, the variable "telephone" ( $95 \%$ true) must be treated as a priority. Awareness campaigns can minimize the impact of this variable. Another action concerns the variable availability of the road (50\% true). Itinerary planning is an example of possible solution. Now once the actions are defined, it remains to anticipate and predict the impact of these actions on the supply function. The main question that arises is why giving preference to this or that action? In order to anticipate decisions and move from an uncertain decision-making environment to a more secure one, influence diagrams are used.

\section{Solution Modeling With Influence Diagram}

The variable that characterizes the logistics, in the Bayesian model, is the merchandise supply. Any decision made must be evaluated in terms of utility. In the logic of influence diagrams, the utility function models the impact of consequences. For our example, this utility function represents the cost of a supply delay, an accident risk, and a break-down risk. For decision-making purpose, utility will be calibrated with respect of the preferences of the manager, or the planner. The circumstances and consequences of the risks vary. They show that risk has two aspects: probability and consequences. For consequences, these are characterized by the supply: availability of merchandise at the customer level. For a general case, it can be the protection of persons, the environment, but also protection of the transport tool or the merchandise itself.

There are two ways to reduce risks: 
- decrease of the probability of occurrence of the undesirable event (non-supply of merchandise),

- attenuation of the consequences of the adverse event,

The influence diagram is an extension of Bayesian networks. An influence diagram, which calculates the utility function measured by a discrete variable, which is the satisfaction of the customer or the supplier of the supplier, plus a decision variable are illustrated by Fig. 4.

Two things are considered here: the decision node "course alteration" to another road like the national road, and the utility node that measures satisfaction. This network also allows the re-evaluation of the supply function with uncertainty measurement. On the influence diagram in Fig. 4, our decision-making is certain, it is $45 \%$, and the probability that the supply of the goods is insured increases to $50 \%$.

There are links from Course alteration and supply of goods to Satisfaction, the ideal is to ensure supply without changing the road (utility $=100$ ), Then when the supply will be sure even with change of road (utility $=90$ ). Finally it is more penalizing if the goods do not arrive in time (utility $=0$ ).

The decision-making studies intend essentially to assess the probability of the occurrence of various risks by taking into account, from the definition of logistic processes and strategies, all the initiating factors:

- technical factors: equipment and transported products (including ergonomics, dangerousness, manufacturing, and quality assurance issues),

- human factors: quality of training especially in safety, ergonomics, qualification,

- environmental factors: natural risks, ambient environments (dust, gas, noise, ...).

Anticipating the impact of decisions has become possible with influence diagrams. In general, if the Bayesian network shown in Fig. 3 models the influence of road risks on merchandise supply, the influence diagram illustrated in Fig. 4 models the decision risk upon the utility function.

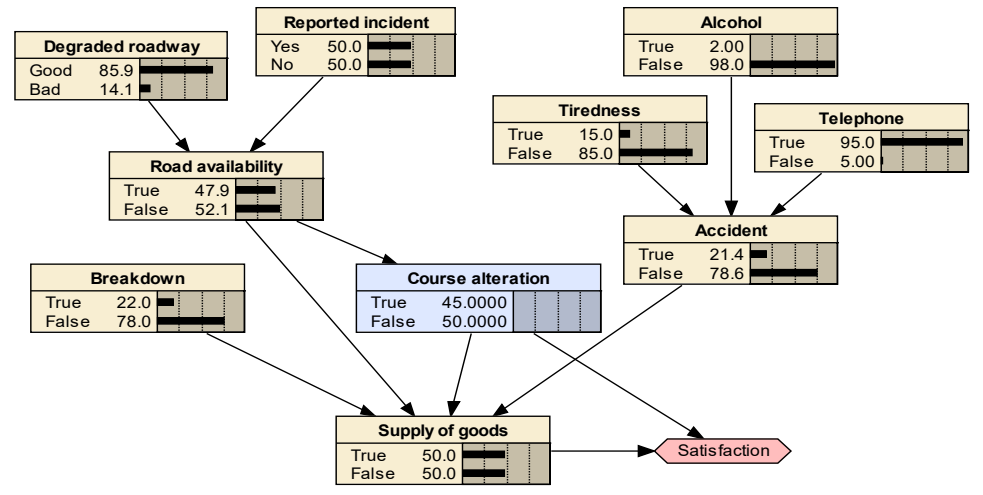

Fig. 4. Influence Diagram generated by Netica.

\section{Conclusion}

In this study, an inventory of the main risks related to the logistic function illustrates the very wide variety of variables for the Bayesian model. This makes the determination of the network structure a step that deserves special attention. A confrontation of experts opinions can figure out this problem, but forgetting a variable will have a serious impact on the results. Logistic activities differ for the majority of them. The simple model, developed in the example of this study, shows the impact of the road unavailability on the supply of the merchandise. 
Through this paper, it is possible to conclude that through surveys, reliable statistics, and experience feedback, it will be possible for the decision-makers and managers, in charge of the logistics function, to make certain decisions. Also, it is possible to select the causes of the most unfavorable risks by characterizing parameters, such as probability of occurrence and severity. The advantage of the model presented in this article is its simple structure, which could be understood by even a non specialist. Another parameter is the intuitive progress (path, evolution, movement) of information (cause - effect). Furthermore, it is extremely important to mention that some (a part) of the studied risks are part of a process leading initially to evaluate the probability of ensuring the supply of merchandise. The prediction tools proposed in this article do not allow, except in the case of redesign of strategy and logistic processes, modifying the incidence of a risk. The presented Bayesian network and influence diagram allow only reducing the severity of the effects induced by a risk on the logistic function. All the risks presented in this article allow carrying out a complete study aiming to improve the logistic processes and to reduce the criticality of risks consequences to an acceptable level. Other parameters, mainly concerning the nature of the merchandise and the behaviour of the driver, can be taken into consideration in the Bayesian model.

\section{References}

1. H.O. Nguyen, J. Tongzon, Transport Policy, 17, 135-146 (2010)

2. W. Hachicha, M. Elmsalmi, Journal of Risk Research, 17, 1301-1324 (2014)

3. A. Toubol, Transport intermodal. Technique de l'ingénieur (AG 8 160v2, 2007).

4. Y. Wu, H. Nakamura, M. Asano, J. Mod. Transport 21, 224-235 (2013)

5. M. Hassan, H. Abdel-Ata, M. Ahmed, A.S. Al-Ghamdi, Transportation Research Part C, 24, 288-298 (2012)

6. B.Uffe, A. Kjærulff, L. Madsen, Bayesian Networks and Influence Diagrams, A Guide to Construction and Analysis (Springer, LLC, 2008)

7. T. Bedford, R. M. Cooke, Probabilistic Risk Analysis: Foundations and Methods (Cambridge University Press, New York, 2001)

8. M.E. Paté-Cornell, Reliab. Eng. Syst. Safe 54, 95-111 (1996)

9. V.M. Bier, L.A. Cox, Advances in Decision Analysis-From Foundations to Applications, Probabilistic risk analysis for engineered systems (Cambridge, 2007).

10. Norsys - Netica Application - Norsys Software Corp. http://www.norsys.com/netica .html

11. P. Damien, Bayesian theory and applications (Oxford University Press, 2015)

12. P. Naim, P.H. Wuillemin, P. Leray, O. Pourret, A. Becker, Réseaux bayésiens. 2 ed. (Eyrolles, France, 2004).

13. A.S. Valladeau, B. Andéol-Aussage, Transport routier de marchandises. Guide pour l'évaluation des risques professionnels (INRS, ED 6095, 2011) 